\title{
The Usefulness of Contrast-Enhanced Ultrasound for Surveillance of Endoleak after Endo-vascular Aneurysm Repair
}

\author{
Shi Jin Cho, Soo Kyung Kwon, Da Mi Jung, Sun Chul Park, Hyang Kyung Kim, Jang Yong Kim, Sang Seob Yun \\ Division of Vascular and Transplant Surgery, Department of Surgery, Seoul St. Mary's Hospital, College of Medicine, The Catholic \\ University of Korea, Seoul, Korea
}

Received October 1, 2019

Revised October 29, 2019

Accepted November 5, 2019

Correspondence to:

Sang Seob Yun

Division of Vascular and

Transplantation Surgery,

Department of Surgery, Seoul St.

Mary's Hospital, College of

Medicine, The Catholic University

of Korea, 222 Banpo-daero,

Seocho-gu, Seoul 06591, Korea

Tel: + 82-2-2258-2876

Fax: + 82-2-595-2822

E-mail: ssyun@catholic.ac.kr
Purpose: Endovascular aneurysm repair (EVAR) is a minimally invasive alternative to open repair for patients with abdominal aortic aneurysm (AAA). Yet the follow-up after EVAR with lifelong post-interventional imaging is mandatory in order to detect complications such as endoleaks or stent migration. Computed tomography angiography (CTA) is considered the standard imaging modality and widely used for follow-up, although patients are exposed to both radiation and nephrotoxic contrast medium. The aim of this study was to determine the diagnostic efficacy of contrast-enhanced ultrasound (CEUS) for detecting post EVAR endoleaks compared with that of CTA, which is considered as the gold standard.

Methods: From January 2010 to August 2019, 27 patients who underwent CEUS and CTA for follow-up after elective EVAR at a single center were retrospectively analyzed. The presence of endoleak was compared between CTA and CEUS.

Results: The 27 paired CTA and CEUS studies were analyzed. Eleven patients had endoleak detected on CEUS and they had the same findings with CTA. One patient had endoleak detected on CEUS, but this was not detected on CTA. Three patients didn't have endoleak detected on CEUS, but endoleak was detected on CTA. 13 patients had no endoleak detected on CEUS or CTA. Assuming CTA is the gold standard, the sensitivity and specificity of CEUS to detect endoleak were $92.3 \%$, and $78.6 \%$ respectively.

Conclusion: CEUS can be used as a viable and feasible imaging alternative for the follow-up and the detection of endoleak after EVAR with the additional benefit of being a real-time non-ionizing radiation examination.

Keywords: Abdominal aortic aneurysm, Contrast enhanced ultrasound, Endovascular abdominal aortic aneurysm repair

\section{INTRODUCTION}

The endovascular aneurysm repair (EVAR) is less invasive therapeutic approach compare to the conventional open abdominal aortic aneurysm repair. (1,

2) EVAR has been accepted as a less invasive procedure with a survival benefit at early post-operative period compared to open repair. However, long-term survival benefit of EVAR over open repair is still unclear, because of its relatively high late complication rate, which make EVAR require lifelong surveillance. $(3,4)$

There are various long-term graft related complications after EVAR, including endoleak, endotension, 
migration, limb kinking, limb occlusion, infection and aortic rupture. (3-5) Endoleak is the most frequent complication after the EVAR, which is defined as the persistence of blood flow outside the lumen of the endoluminal graft within the aneurysmal sac. The existence of endoleak could lead to enlargement of aneurysmal sac and increase the risk of aneurysm rupture.(1) Thus, there are some guidelines recommending regular follow up after EVAR.(3-5) The computed tomography angiography (CTA) has been a preferred surveillance tool for a routine follow up despite of the radiation exposure and nephrotoxic iodinated contrast media. $(3,4,6)$ To reduce those additional risk, the contrast enhanced ultrasonography (CEUS) using non-nephrotoxic sulphur hexafluoride microbubbles as contrast has been developed and gained acceptability as post-EVAR surveillance in recent years. (7-9) This study was aimed to elucidate the accuracy of CEUS compared to CTA regarding the detection of endoleak during post-EVAR follow-up.

\section{METHODS}

This is a retrospective study of 155 patient who undergone EVAR from January 2010 to February 2019. 38 patients undergone follow-up surveillance after EVAR who had received at least one CEUS examination after the stent implantation were investigated. Among 38 patients, 10 patients who underwent CEUS only without CTA were excluded. One patient experienced AAA rupture before routine surveillance was excluded. The result of the CEUS was compared with those of the CTA, which was considered to be the 'gold standard'.

The CEUS images were obtained using a duplex ultrasound instrument (Philips IU22, Washington, USA or General Electric E9, Milwaukee, USA) with the C5-1 curved array transducer performed by an experienced sonographer. Once the unenhanced ultrasonographic protocol is completed, a SonoVue bolus injection, consisting of stabilized microbubbles of sulfur hexafluoride, is administered intravenously for the CEUS examination. 2.4 mL of sonoVue once a time, maximum dose up to $4.8 \mathrm{~mL}$, was injected into an antecubital vein through a 20-gauge needle and was followed by a flush of $5 \mathrm{~mL}$ saline solution $(0.9 \%$ $\mathrm{NaCl}$ ) Administration of $2.4 \mathrm{~mL}$ contrast agent allows diagnostic views for about 3 minutes. After $5 \mathrm{mi}-$ nutes, almost all of the contrast has vanished. During the period, sonographer evaluate whether blood flow exist inside the aneurysmal sac. Both coronal view and longitudinal view are assessed. If endoleak is detected, then origin of the blood flow is evaluated to assess entoleak type. This series of procedures should be carried out in about three minutes. If needed second dose of $2.4 \mathrm{~mL}$ can be administered.

All patients had paired CTA images obtained on a helical scanner with a $1 \mathrm{~mm}$ slice thickness (Siemens Sensation, Siemens Medical, Germany). CTA from the diaphragm to femoral heads was performed with the patient in the supine position. A dose of $120 \mathrm{~mL}$ of the iodinated contrast medium Iomeron 350 (Iomeprol, $350 \mathrm{mg} / \mathrm{mL}$ ) was administered at a flow rate of $4 \mathrm{~mL} / \mathrm{s}$. The imaging took about 15-20 minutes.

Statistical analysis was performed with the non-parametric exact binomial test, the significance level was tested at 0.05 . The concordance between the two tests was also analyzed using the kappa coefficient. Statistical analysis was performed using IBM SPSS Advanced statistics version 24 (IBM, Armonk, NY, USA).

\section{RESULTS}

The study enrolled 27 patients who undergone CEUS and CTA after EVAR. 21 were male (77.8\%) and 6 were female with mean age of $75.5 \pm 9.4$ years (range, 51-90 years). The mean follow-up period was $42.0 \pm 30.3$ months (range 3-116 months). Radiological appearances of endoleak on CTA and 
CEUS is shown in Fig. 1 and 2, respectively.

The exact binomial test showed no significant difference between the result of the CEUS and those of the CTA (P-value > 0.05). Also, kappa coefficient test of the CEUS comparing to the CTA showed the results of the two methods show substantial coincidence $(\kappa=0.705)$. The result of the CTA and the CEUS is shown in Table 1. Assuming CTA ia a gold standard, the sensitivity, specificity of CEUS to detect endoleak were $92.3 \%$, and $78.6 \%$, respectively.
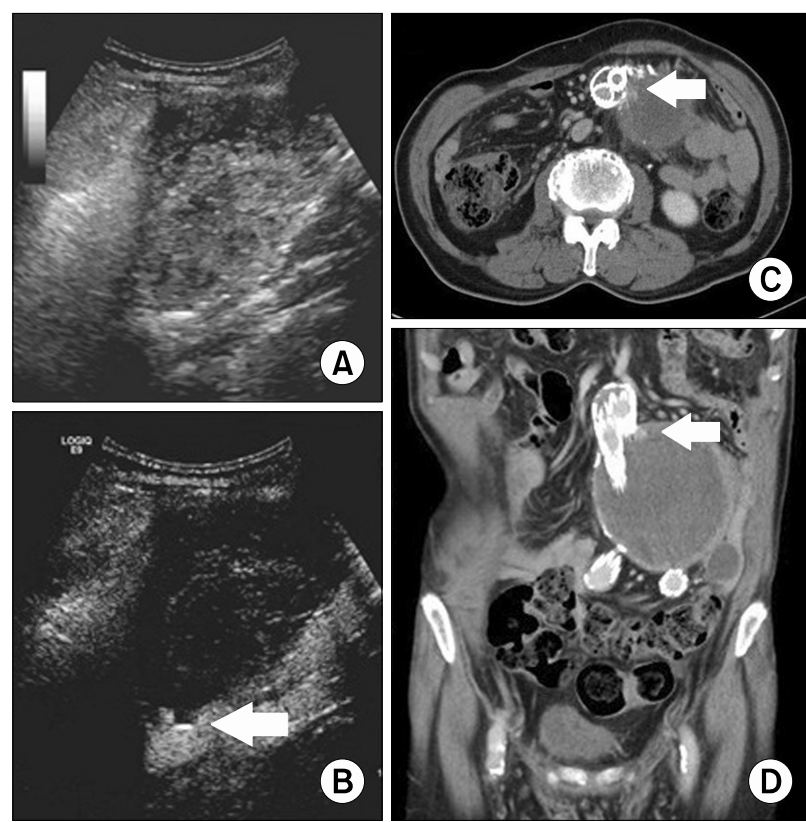

Fig. 1. Type I endoleak is shown in (A) conventional B-mode, (B) CEUS, (C) and (D) CT angiography. Extravascular enhancement is shown (arrow) within the aneurysm sack.
Table 2 shows more detailed information of the patients who were diagnosed with endoleak by either CTA or CEUS. Type of the endoleak is also evaluated. 7 patients are evaluated for identical type of endoleak, and 8 patients aren't.

\section{DISCUSSION}

EVAR is getting more proportion as the surgical treatment of the AAA than that of the open repair, because of the lower burden of cardiopulmonary complications and the lower short-term mortality rate. (10) But long-term survival and reintervention rate after EVAR is not superior to that of the open surgery resulting from endoleak or stent migration. (2) Because of these stent-related complications, there are a few guidelines for post-EVAR routine surveillance obviously insisting more frequent imaging studies than after the open repair.(3-5) Traditionally, annual CTA was the gold standard imaging study for the post EVAR patients. $(11,12)$ However,

Table 1. Endoleaks Detected with Computed Tomography Angiography (CTA) and Contrast-Enhanced Ultrasound (CEUS) Imaging

\begin{tabular}{llcc}
\hline & \multicolumn{2}{c}{ CTA } \\
\cline { 3 - 4 } & & Endoleak & No endoleak \\
\hline \multirow{2}{*}{ CEUS } & Endoleak & 11 & 1 \\
& No Endoleak & 3 & 12 \\
\hline
\end{tabular}
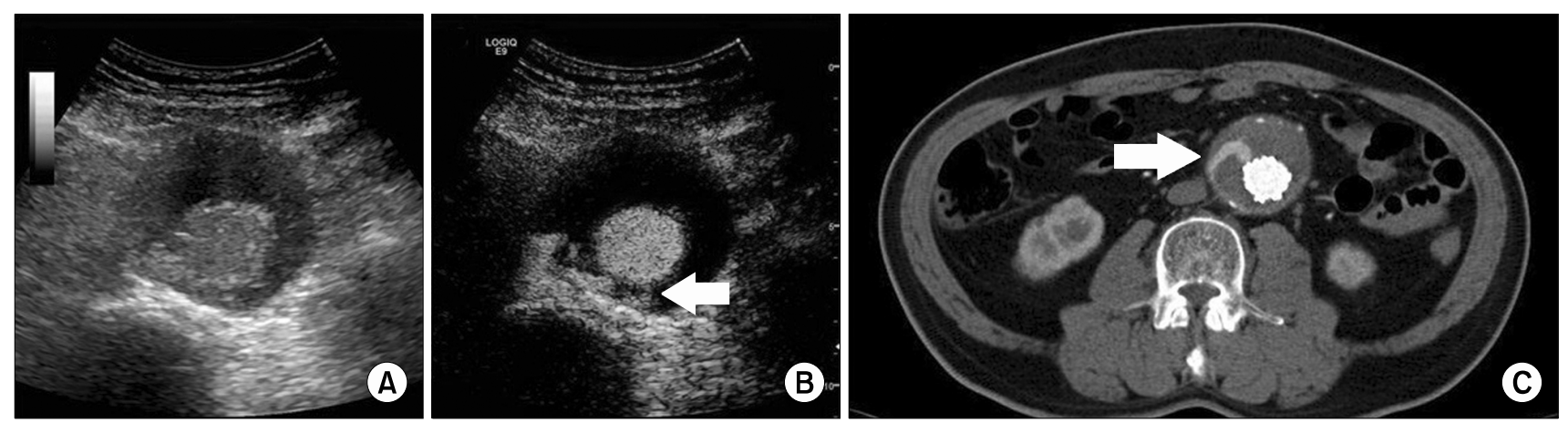

Fig. 2. Type II endoleak from a lumbar artery is shown in (A) conventional B-mode, (B) CEUS, (C) CT angiography. Extravascular enhancement is shown (arrow) within the aneurysm sack. 
Table 2. Results of the Computed Tomography Angiography (CTA) and Contrast-Enhanced Ultrasound (CEUS) Imaging

\begin{tabular}{ccccccc}
\hline Patient No. & Sex & Age & CTA & CTA endoleak type & CEUS & CEUS endoleak type \\
\hline 1 & $\mathrm{M}$ & 79 & Endoleak & 1 & Endoleak & 1 \\
2 & $\mathrm{M}$ & 84 & Endoleak & 1 & Endoleak & 1 \\
3 & $\mathrm{M}$ & 82 & Endoleak & 1 & Endoleak & 1 \\
4 & $\mathrm{M}$ & 82 & Endoleak & 1 & Endoleak & 2 \\
5 & $\mathrm{M}$ & 82 & Endoleak & 1 & Endoleak & 3 \\
6 & $\mathrm{M}$ & 76 & Endoleak & 2 & Endoleak & 1 \\
7 & $\mathrm{M}$ & 82 & Endoleak & 2 & Endoleak & 2 \\
8 & $\mathrm{M}$ & 68 & Endoleak & 2 & Endoleak & 2 \\
9 & $\mathrm{~F}$ & 72 & Endoleak & 2 & Endoleak & 2 \\
10 & $\mathrm{~F}$ & 86 & Endoleak & 2 & Endoleak & 2 \\
11 & $\mathrm{~F}$ & Endoleak & 3 & Endoleak & 2 \\
12 & $\mathrm{M}$ & No endoleak & - & Endoleak & 2 \\
13 & $\mathrm{~F}$ & 84 & Endoleak & 1 & No endoleak & - \\
14 & $\mathrm{M}$ & 51 & Endoleak & 2 & No endoleak & - \\
\hline
\end{tabular}

recent guidelines recommended ultrasonography as the substitute for CTA because it entails radiation exposure and nephrotoxic contrast injection. $(6,9,13)$

In this study, detection of the endoleak using CEUS showed sensitivity and specificity of $92.3 \%$, and $78.6 \%$ respectively. Moreover, the results between CEUS and CTA are coincidence $(\kappa=0.705)$. The result of this study showed that results of the CEUS coincided with those of the CTA which implies that CEUS may substitute for CTA as a routine surveillance tool after EVAR.

Four patients showed inconsistent results for the presence of endoleak between CTA and CEUS. First patient has shown aneurysmal sac enlargement and type II endoleak from the lumbar artery by CEUS. Follow-up CTA was done after 3 months, and the endoleak was self-remissioned. Second patient underwent negative CEUS a year before to the compared CTA which detected type 1 endoleak. In these two cases, the time gap between the compared imaging studies interferes accurate comparison which is the limitation of this study result from its retrospective nature. The other two patients have shown minimal curvilinear enhancement around proximal portion of the stent by the CTA, which is suspicious feature of minimal endoleak. Follow-up CEUS revealed no sign of endoleak.

CEUS doesn't only detect existence of the endoleak but also evaluates the type of endoleak. Type of endoleak is the one of the most important factors to decide re-intervention after EVAR. Endoleak type, increase of aneurysmal diameter, endoleak size are assessable with CEUS. CEUS might be superior to the CTA detect type of endoleak in aspect of its nature of real time evaluation. But it is hard to say superiority of CEUS compare to CTA in this study because patient with endoleak didn't underwent diagnostic angiography.

The risk factors of the AAA includes old age, atherosclerosis, cerebrovascular disease, coronary artery disease, hypercholesterolemia, hypertension etc. (14) And these factors are also risk factors for the chronic renal disease (CKD). (15) There were 10 patients only done CEUS after EVAR because of elevated creatinine level, whom were excluded from this study model by reason of lacking of comparable "gold standard study". The benefit of routine surveillance with CTA after EVAR are done every year to evaluate existence of endoleak in patient with CKD are not proven, and guidelines are recommending alternatives such as 
non-enhanced computed tomography, ultrasonography. $(3,5)$

It seems hard to draw firm conclusion from small sample size. Still it could be a pilot study for evaluating CEUS as a tool for post-EVAR patients. Further anterograde large-scale study would be needed for expiscating the accurate value and the limitations of the CEUS comparing with CTA.

Although the benefit of the CEUS compared to the CTA are clear, also it has several limitations. $(8,9)$ First, the result of the CEUS, relies on sonographer's techniques. Which infers skillful sonographer is needed and requirements of considerable learning curve for the trustworthy examinations. Which may limit implementation of CEUS in non-tertiary and low volume hospitals. Second, some of patient characteristics, such as obesity (BMI > 30), gaseous abdominal distension, and ascites, can obstruct accurate examinations by making poor acoustic window.

\section{CONCLUSION}

CEUS is a fast, minimally invasive, portable and reliable method for detecting endoleak during the surveillance after EVAR. Moreover, the CEUS could be a good substitute of CTA for the patient who are prohibited for radiation exposure and injection of nephrotoxic contrast medium.

\section{REFERENCES}

1. Patel R, Sweeting MJ, Powell JT, Greenhalgh RM. Endovascular versus open repair of abdominal aortic aneurysm in 15-years' follow-up of the UK endovascular aneurysm repair trial 1 (EVAR trial 1): a randomised controlled trial. Lancet 2016;388:2366-74.

2. Patel R, Powell JT, Sweeting MJ, Epstein DM, Barrett JK, Greenhalgh RM. The UK endovascular aneurysm repair (EVAR) randomised controlled trials: long-term follow-up and cost-effectiveness analysis. Health Technol Assess 2018;22:1-132.

3. Wanhainen A, Verzini F, Van Herzeele I, Allaire E, Bown M, Cohnert T, et al. Editor's choice - European
Society for Vascular Surgery (ESVS) 2019 clinical practice guidelines on the management of abdominal aorto-iliac artery aneurysms. Eur J Vasc Endovasc Surg 2019;57:8-93.

4. Zierler RE, Jordan WD, Lal BK, Mussa F, Leers S, Fulton J, et al. The Society for Vascular Surgery practice guidelines on follow-up after vascular surgery arterial procedures. J Vasc Surg 2018;68:256-84.

5. Chaikof EL, Dalman RL, Eskandari MK, Jackson BM, Lee WA, Mansour MA, et al. The Society for Vascular Surgery practice guidelines on the care of patients with an abdominal aortic aneurysm. J Vasc Surg 2018;67:2-77.e2.

6. Mitchell AM, Jones AE, Tumlin JA, Kline JA. Incidence of contrast-induced nephropathy after contrast-enhanced computed tomography in the outpatient setting. Clin J Am Soc Nephrol 2010;5:4-9.

7. Zimmermann H, D'Anastasi M, Rjosk-Dendorfer D, Helck A, Meimarakis G, Reiser M, et al. Value of high-resolution contrast-enhanced ultrasound in detection and characterisation of endoleaks after EVAR. Clin Hemorheol Microcirc 2014;58:247-60.

8. Bredahl KK, Taudorf M, Lönn L, Vogt KC, Sillesen H, Eiberg JP. Contrast enhanced ultrasound can replace computed tomography angiography for surveillance after endovascular aortic aneurysm repair. Eur J Vasc Endovasc Surg 2016;52:729-34.

9. Chisci E, Harris L, Guidotti A, Pecchioli A, Pigozzi C, Barbanti E, et al. Endovascular aortic repair follow up protocol based on contrast enhanced ultrasound is safe and effective. Eur J Vasc Endovasc Surg 2018; $56: 40-7$.

10. Paravastu SC, Jayarajasingam R, Cottam R, Palfreyman SJ, Michaels JA, Thomas SM. Endovascular repair of abdominal aortic aneurysm. Cochrane Database Syst Rev 2014;(1):CD004178.

11. Stavropoulos SW, Charagundla SR. Imaging techniques for detection and management of endoleaks after endovascular aortic aneurysm repair. Radiology 2007; 243:641-55.

12. Chaikof EL, Brewster DC, Dalman RL, Makaroun MS, Illig KA, Sicard GA, et al. SVS practice guidelines for the care of patients with an abdominal aortic aneurysm: executive summary. J Vasc Surg 2009;50:88096.

13. Jones C, Badger SA, Boyd CS, Soong CV. The impact of radiation dose exposure during endovascular aneurysm repair on patient safety. J Vasc Surg 2010; 52:298-302.

14. Keisler B, Carter C. Abdominal aortic aneurysm. Am Fam Physician 2015;91:538-43.

15. Ahmed SB, Dumanski SM. Why do patients with well-controlled vascular risk factors develop progressive chronic kidney disease? Can J Cardiol 2019; 35:1170-80. 